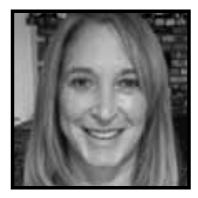

\title{
Nurturing Students' Brains for the Future
}

Judy Willis

\section{ABSTRACT}

As we strive to build a better world for our children and better prepare our students for the complexities of the globalized world they will enter, neuroscience research can be correlated with educational strategies to help us build students' brains to meet that future. An example of the nexus of research and teaching comes from correlating implications from research about memory, cognition, neuroplasticity, and discoveries about the timing of brain maturation with the incorporation of transfer opportunities throughout instruction, such that students apply learning to contexts beyond those in which it is taught and rehearsed.

\section{The Highest Brain Processes Most Actively Develop During the School Years}

he most complex thinking, reasoning, and innovation-promoting region
of the brain is the prefrontal cortex (PFC). Within the PFC are the brain
circuits of executive functions. These control networks directing decision making and self-control are the last part of the brain to mature, undergoing their most rapid maturational changes during the school years.

Maturation in the brain refers to the pruning away of unused neurons and their connections and to the neuroplasticity that strengthens neural networks that are more frequently activated by use. This PFC maturation continues well into the twenties, making teachers critical nurturers of the development of the part of the brain that distinguishes our humanity and defines the qualities students bring with them into their adult lives. 
The executive function networks, of neurons connecting together via their branching axons and dendrites, are the control centers of conceptual thinking and highest cognition including critical analysis, judgment, prioritizing, organizing, openmindedness/flexibility, collaboration skills, induction, deduction, creative/innovative problem solving, and delay of immediate gratification for long-term goal development. It is these highest cognitive, social, and emotional skill sets that are needed for the brain to analyze and utilize the increasingly expanding volume of information that awaits our students in their futures.

Neuroscience knowledge can offer insights to help us guide students' prefrontal cortex development while we also contend with the demands that they "learn" an already over-stuffed curriculum that keeps expanding beyond appropriate teaching limits in response to more available information. Anyone involved with administration or classroom instruction knows that promoting students' rote memory of an over-stuffed curriculum is inadequate preparation for them to build the skill sets they will need to accurately assess, as well as innovatively use, the growing surge of new information and changing "facts" (remember when Pluto was a planet?).

As we strive to prepare students for the $21^{\text {st }}$ century, with problems and opportunities not yet recognized, the development of executive functions such as judgment, flexibility, and critical analysis take on even greater significance. Before Copernicus recognized that the earth revolves around the sun, the accepted "fact" was that the sun orbited the earth. Students need to have guided experiences evaluating validity of information and applying learning to tasks beyond their original learning framework to be prepared to take optimal advantage of the current and future expanding funds of knowledge and changing "facts."

Consider that 30 percent of today's jobs did not exist 20 years ago. Success in jobs of the future will not seek out candidates with the limited perspective of rote memorization of the increasing volume of facts, when facts as we now know them will be revised or disproved in the future.

If students leave school without the skill sets of executive function activated during their critical prefrontal cortex maturation years, their expanded volume of memorized facts will be inadequate preparation for the challenges and opportunities of the $21^{\text {st }}$ century. They will not be prepared to enter careers where they will need to apply executive functions to evaluate, understand, and innovatively apply the advances in technology and global communication to the exponentially growing information base. 


\section{Preparing Brains for the Future With Transfer Opportunities Now}

The developing neural networks of executive functions (EF) in the prefrontal cortex can and must be activated and strengthened by use if students are to reach their maximal potentials for these highest cognitive, social, and emotional skill sets. Teaching children while the PFC is undergoing its most rapid development from ages 8-18 is challenging when rote memorization takes up much instruction time. However, you can promote EF activation by incorporating, into learning and assessment, opportunities that allow students to process, evaluate, and ultimately transfer new information and procedures to applications beyond those in which they are learned.

Transfer opportunities, embedded in curriculum, assessment, and lesson instruction build up neural networks of memory storage throughout the brain's cortex and also exercise the developing networks of executive functions in the prefrontal cortex. Transfer of learning to novel situations provides opportunities to mentally manipulate new learning to solve new types of problems. Through neuroplasticity, transfer activities extend the boundaries of memory storage beyond isolated circuits in which the brain holds information that is only passively learned and drilled into memorized facts.

When a memory circuit is activated, information is conducted from neuron to neuron by electrical flow. Neuroplasticity strengthens active networks in response to their increased electric flow. The components of neuroplastic growth include thicker myelin insulation around the axons that carry information from each neuron on to the next and more connections among the memory circuit's neurons (more dendrites and synapses). These changes result in faster speed of information transfer, more rapid memory retrieval, and greater memory durability so the network is less susceptible to the pruning of disuse.

In addition to stimulating the neuroplastic development of the rapidly maturing prefrontal cortex networks, transfer tasks can increase long-term conceptual memory storage. Transfer learning opportunities can be designed so students use executive functions and activate previously unrelated memory circuits of facts for use together in new applications. This simultaneous stimulation, of previously unconnected circuits of stored information, promotes growth of connections among these circuits as the neurons that fire together, wire together. 
Simultaneous activation of previously isolated memory circuits (such as rote memorization of mathematics formulas or content-specific vocabulary words) for use with other memory networks to perform new tasks promotes the neuroplastic changes that incorporate the rote memory circuits into larger circuits. These interconnections transform the isolated memories, which previously were only activated by the specific stimuli with which they were learned and practiced, into relational concept memory circuits.

Through incorporation of new learning into the larger related neural networks of concepts, the new learning will not become an isolated, unused neural network that will be pruned away after test prep is over. Connected relational concept networks become the expanded funds of applicable wisdom that executive functions can access far beyond the school years. These memory concept networks, along with the strong circuits of the executive functions needed to apply the knowledge they hold, are the skill sets needed for their future critical analysis, creative problem solving, and innovation. The physical construction of the networks of executive functions and conceptual memories will prepare today's students to solve problems not yet revealed and benefit from the opportunities that will be provided by information and technology not yet developed.

\section{Motivating Engagement and Perseverance}

When students know the information they are being asked to learn will be used to create products or solutions to problems that interest them, the new learning and its practice are valued because they want to know what they have to learn. The expectation that new learning will be applied to desired goals increases the strength of memories through their association with positive emotions. Transfer tasks, that are planned so students can engage through strengths and interests to achieve goals they consider relevant, are powerful learning motivators and memory enhancers.

An example of transfer for the multiple purposes of motivation, conceptual long-term memory, and executive function mental manipulation is project-based learning with multiple options of approach and solutions. To promote the requisite learning goals and the activation of the rapidly changing networks of executive function, transfer activities should be designed to require several of these functions such as judgment, prioritizing, organizing, communication, collaboration, induction, deduction, prior knowledge activation, and/or prediction. These learning experiences 
then serve to motivate participation and to promote critical analysis and goal-planning as well as flexible, divergent thinking.

Cross-curricular project-based learning further increases the opportunities for transfer and student motivation. Robotic design is an example using physics, biology, engineering, math, Internet research, economics, language arts, and graphic arts that also incorporates real world problems, student interests, and offers a variety of opportunities to contribute through individual strengths in collaborative group projects. Robotic limb replacement or mechanical enhancement of healthy arms and legs could link the need for such devices by people who have lost limbs in battle (current events/history) with student interest in sports. Students can choose to work on robotic limb replacements that could theoretically provide physical enhancements relevant to their own high interest sports, such as jumping higher to get more "air time" for skateboard or snowboard maneuvers.

\section{Teachable Moments Into Transfer Tasks}

The concerns and complaints of students can be noted and subsequently incorporated into projects using transfer of learning. For example, students may voice their frustration regarding the extra time it takes to get to school because of a series of new traffic lights. A look at upcoming units of study might include causes and interventions regarding pollution in science and the solving of single variable equations $(3 X+1=7)$ in math. A project can be planned that connects the required learning with students' real life concerns. They could be given the opportunity to use the requisite math and science to accumulate data and formulate interventions to achieve their personally desired goal (shorter drive time) and a desirable community goal of cleaner air.

Some students may predict that the timing of traffic lights can be synchronized to reduce idling time of the greatest numbers of cars, limit delay, and reduce polluting emissions. They might further consider the requirements for adequate frequency and duration of "go" time at these intersections for safe crossing by pedestrians of all ages and physical abilities.

Because project tasks are planned in advance, such that students apply learning from the unit of study as they investigate and plan, the information they are required to learn becomes knowledge they want to acquire. Now they approach 
instruction with personal interest as they value the knowledge. The varied options and new applications of the ongoing learning provided by the transfer to the new applications of the project thereby increases motivation while promoting executive function activation.

Further authenticity of goals, along with opportunities for students to build literacy and communication skills, are promoted when transfer tasks are designed to include interviews with experts, especially those with different perspectives so they can exercise their information analysis. Motivation is enhanced from preparation of project information for an authentic "audience" such as a proposal for local government in writing or at a public hearing, letters to the local newspaper editor, or presentations to invited professionals who come to the class to hear, view, and respond to students' proposals.

\section{Feedback}

As a survival imperative, the mammalian brain responds with increased attention to feedback about the accuracy of predictions/choices it makes. Further, through a dopamine-prediction reward circuit, this feedback results in increased memory consolidation. Ongoing formative assessment and feedback by teachers, teacher-guided peer feedback, and opportunities for self-reflection and revision further promote executive function activation such as judgment and goal-directed behavior.

Transfer activities should be planned to offer students feedback about the outcomes of their predictions as well as opportunities to recognize the positive relationship between their effort and goal-progress. The amount of guidance, scaffolding, or increased challenge provided in the feedback can be varied as a way of differentiating for the range of student needs or mastery.

Giving students' networks of executive functions cognitive workouts, during their most stimulation-responsive growth phase, is both the privilege and responsibility of all educators. The nurturing of these cortical circuits during their most active developmental phase impacts the cognitive resources students bring with them into adulthood. Today's classroom interventions are the powerful neurological tools with which we can help all students embark on their futures with their highest potentials for social, emotional, and professional fulfillment. 


\section{The Future}

Transfer learning experiences that incorporate executive functions will go far to promote students' "higher processing" as they construct the powerful concept networks needed for accurate and flexible analysis and utilization of future information. Students' concept-constructed long-term memory may first be evident in their more accurate "predictions," such as response to test questions that are not identical to the homework problems they practiced.

These same brain networks will grow and ultimately guide these students to make the wisest choices in work and social situations, and ultimately to use creative innovation to find the most successful solutions to future problems. These students will have the neural architecture and experience to solve the problems as well as participate in the opportunities that await them in the $21^{\text {st }}$ century.

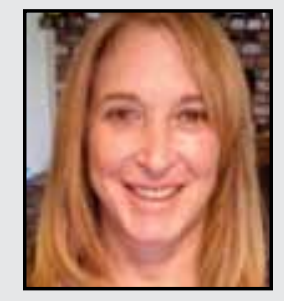

Judy Willis graduated Phi Beta Kappa as the first woman graduate from Williams College and attended UCLA School of Medicine. Dr. Willis remained at UCLA and completed a medical residency and neurology residency, including chief residency. She practiced neurology for 15 years before returning to university to obtain her teaching credential and master's of education from the University of California, Santa Barbara. She then taught in elementary and middle school for 10 years and currently provides professional development presentations and workshops nationally and internationally about learning and the brain.

LINK TO:

http://www.RADTeach.com 